\title{
Determination of technological parameters and tool for flanging holes from sheet blanks
}

\author{
Khaidar Kaderov ${ }^{1 *}$, Sergey Kireev ${ }^{1}$, Marina Korchagina ${ }^{1}$, and Aleksey Lebedev ${ }^{1}$ \\ ${ }^{1}$ Don State Technical University, sq. Gagarina, 1, Rostov-on-Don, 344010, Russia
}

\begin{abstract}
In the article the parameters of shaping operations of internal axisymmetric cylindrical flanging are considered. The article is written to improve the quality and speed of flanging round holes development from sheet blanks and tooling construction by increasing the accuracy of hole diameter calculation for flanging in the automated mode of building threedimensional models of workpiece and a flanged product. Given formulas increase the accuracy of hole diameter calculation for flanging, taking into account the deformed condition of the sheet blank. The accuracy of the hole diameter calculation for flanging according to the given formulas is determined by comparing the mass of the workpiece with the mass of the flanged part. CAD system algorithm for automated construction of the flanged parts sketch and determination of technological parameters is implemented. The parameterized scheme of a typical construction of a flanging stamp is presented. The constructed schemes allow making changes in their parameters and providing automatic representation of calculation results and also their correct graphic interpretation. Discussion of the article results allows drawing conclusions about correctness of the given mathematical model and its applicability for the automated construction of three-dimensional models of the flanged details that will allow simplifying technological development and constructed documentation with simultaneous increase of their accuracy and reducing costs of production preparation.
\end{abstract}

\section{Introduction}

Modern technologies of metal processing by means of pressure differ significantly from those used several decades ago. For example, heat rays are widely used for cutting contours. For a number of form-changing operations, processing centers are developed, controlled by the corresponding computer applications. However, this equipment is very expensive: from several hundred thousand to several million dollars. They can be purchased by large firms, and for small and medium-sized firms this price is unacceptable. In addition, it should be noted that the country has a fleet of pressing equipment, which continues to be replenished. Keeping it in mind, we can conclude that the development of technology and construction of tooling continues to be relevant.

The aim of the article is to improve the quality and accelerate the development of

*Corresponding author: spu-58.2@donstu.ru 
technology and construction of tooling for flanging round holes from sheet blanks. To do this, it is necessary to improve the accuracy of calculating the dimensions of workpiece, automate the definition of the main technological parameters and the construction of the appropriate tool. The solution of the tasks will simplify the development of technological documentation and reduce the cost of production preparation.

The main technological parameters of forming operations of sheet stamping include:

1. Dimensions of workpiece.

2. The coefficients of the deformation.

3. Power parameters required for the operation.

4. Equipment selection.

Development of technological equipment for sheet stamping includes the following stages:

1. Selection of the tooling constructed scheme.

2. Method of workpiece positioning.

3. Design selection and tool sizing.

4. Specification of equipment parameters.

The task is to achieve this goal and to build a parametric model in CAD system, which allows making changes of the model parameters and providing automatic representation of the calculations' results and their correct graphical interpretation.

\section{Materials and methods}

The implemented aim is as follows:

1. Improving the accuracy of hole diameter calculation for flanging achieved by creating a mathematical model of the deformed state of workpiece during shaping, based on compliance with change conditions in metal thickness in the deformation center and the constancy of the volume.

2. The solution of automation problem of the main technological parameters determination of flanging and constructing of the corresponding tool is realized with the use of CAD system resources.

The existing methods for determining the diameter of the technological hole proceed from the condition that the height of the side in the products does not exceed 0.3 of the selected hole diameter [1]. On the basis of it, the authors [2] proposed to calculate the diameter of the hole by analogy with the bending operation: the length of the side sweep to equate the width of the annular deformable portion of workpiece (Fig. 1)

$$
d_{h}=D+2,4 s+0,9 r_{m}-2 h
$$

The specified [2] calculation condition of hole diameter is also accepted (Fig. 2)

$$
d_{h}=D-2\left(H-0,43 r_{m}-0,72 s\right)
$$

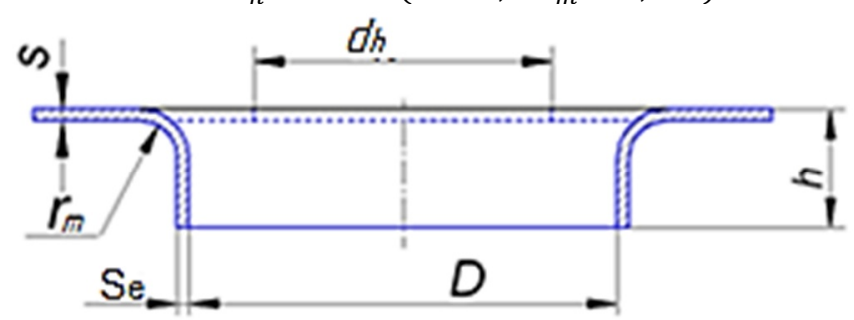

Fig 1. Initial data for the calculation of the flanging hole diameter for formula (1) 


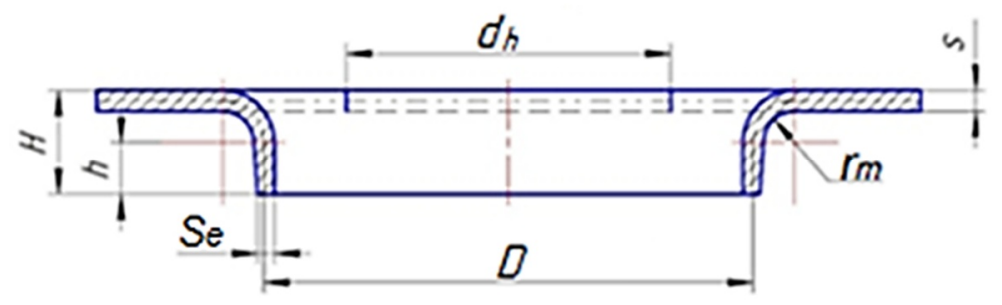

Fig 2. Initial data for the calculation of the flanging hole diameter for formula $(2,3,4)$

It should be noted that in the formula (2) the diameter of the flanging $D$ refers to the middle surface.

The condition of length equality of side sweep is wider than of the deformed ring site of work piece (Fig. 2) applied by prof. Popov E. A. (according to Technology and automation of sheet stamping. Moscow, BMSTU Publ.) in the derivation of the formula

$$
d_{h}=D-0,57 s-1,14 r_{m}-2 h,
$$

where the size $D$ refers to the median surface and $h$ is the length of the cylindrical portion of the side.

In other sources, for high sides and small values of hole diameter, it is proposed to calculate the diameter of the holes for flanging from the condition of equality of initial workpiece volumes and the deformed workpiece [3]

$$
d=\sqrt{D_{1}^{2}-2 \pi D_{1}\left(r_{1}+\frac{s}{2}\right)+8\left(r_{1}+\frac{s}{2}\right)^{2}-4\left(D_{1}-2 r_{1}-s\right)} h
$$

where $h$ is the length of the cylindrical part of the side, $r 1$ is the outer radius of the rounding (corresponds to the $r_{m}$ in Fig. 2) $D l=D+2 r_{l}, D$ - diameter of the middle cylindrical surface of the side. However, even here, the change in metal thickness in the deformation center is not taken into account.

Thus, in any formula from the specified (1-4) change of metal thickness in the deformation center is not considered. At the same time, sources [4 - 6] note the thinning not only of the edge of the side, but also the change in thickness in the entire deformable part of the workpiece in accordance with the stress-strain state of the deformation center. The thickness of the side edge sc is determined from the ratio

where $-c_{f l}$ the coefficient flanging.

$$
s_{c}=s \sqrt{c_{f l}},
$$

It should be noted that prof. Popov E. A. (according to Technology and automation of sheet stamping. Moscow, BMSTU Publ.) proposed a formula that allows us to calculate the effect of changes in metal thickness during flanging of deformations in the meridional direction

$$
\Delta L_{f}=\Delta L_{i}\left(\frac{\rho_{l}}{R_{s}}\right)^{\frac{2 r_{0}-\rho}{r_{0}+\rho}},
$$

where $L_{f}$ and $L_{i}$ - final and initial dimensions of the workpiece in the meridional direction, $\rho_{\mathrm{i}}$ and $\rho_{\mathrm{f}-}$ the initial and final distances of the considered element from the axis of symmetry $\left(\rho_{f}=R_{s}\right), R_{\mathrm{s}}$ - the initial and final distances of the considered element from the axis of symmetry, $\rho$ - the current radius of the element.

In the articles $[7,8,9]$ it is shown in detail, on specific examples, that the results of calculations of hole diameter for flanging according to the above formulas have significant differences among themselves. At the same time, as stated, the condition of volume constancy is not followed, what does not allow building a three-dimensional model of the workpiece and using modern methods to analyze its stress-strain state in the deformation process and assess the strength of finished products.

It should be noted that these formulas were proposed in the 60's-70's of the last century 
with significant simplifications, when computer technology was not practically used. Taking into account all the above, it was proposed to take into account the deformed condition during shaping and the condition of volume constancy when determining the diameter of the hole.

Researches of thin-walled axisymmetric workpiece forming (at the ratio of wall thickness to diameter $s / D \leq 5 \%$ ) from sheet blanks is singled out [10]: the modules of the main logarithmic deformations with sufficient accuracy can be determined with the formula (5) to define the thickness of the flanging edge.

$$
\left|\varepsilon_{1}\right| \approx 2\left|\varepsilon_{2}\right| \approx 2\left|\varepsilon_{3}\right|
$$

where is the deformation: $\varepsilon_{1}$ - rounding, $\varepsilon_{2}$ - formed along, $\varepsilon_{3}$ - by thickness.

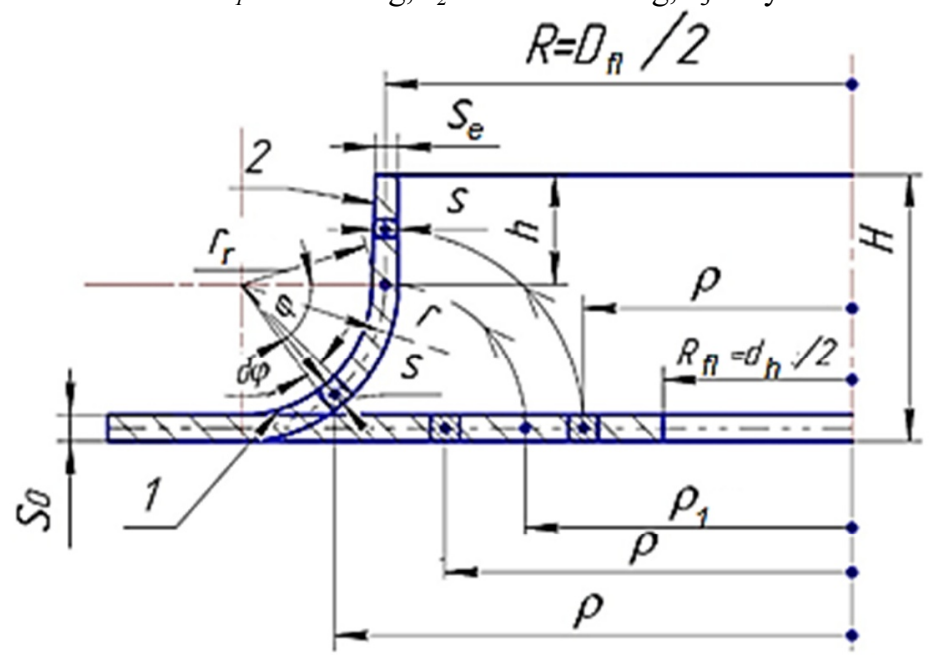

Fig. 3. Scheme for hole diameter calculating for flanging

$D_{f l}$ - diameter of the middle surface, $H$ - full side height, $h$ - height of the cylindrical section of the side; $s_{0}$ - the original thickness of the sheet, $r_{r}$ - radius of rounding; $r$ - radius of rounding of the middle surface; $\rho$ - current radius; $\rho_{1}$ - radius ensuring the formation of radius of the part; $d \varphi$ - the increment of the angle; $s_{e}$ - side edge thickness

Let us write down the equality of the elementary radius section volumes and the corresponding section of the flat ring (see Fig. 3)

Taking into account that

$$
2 \pi r[R+r(1-\cos \varphi)] s d \varphi=2 \pi s_{0} \rho d \rho
$$

$$
s=s_{0} \sqrt{p /[R+r(1-\cos \varphi)]},
$$

where $\rho$ - current radius of the original planar ring, $d \rho$ - corresponding to the elementary ring, $d \varphi$ - on the 1 -st section, and by reducing both parts to the $2 \pi s_{0} \sqrt{\rho}$, we can get

$$
r \sqrt{R+r(1-\cos \varphi)} d \varphi=\sqrt{\rho} d \rho
$$

The equation (10) can be represented as

$$
r \sqrt{R} \sqrt{1+(r / R)(1-\cos \varphi)} d \varphi=\sqrt{\rho} d \rho
$$

The integration of both parts of the equation was performed. The meaning of the integral

$$
\int_{0}^{\pi / 2} \sqrt{1+(r / R)(1-\cos \varphi)} d \varphi
$$

found numerically, with the ratio $\frac{r}{R} \epsilon \bar{r}$ changed from 0.05 to 1,0 in increments of 0.05 According to the results of calculations, the meaning of the integral (12) for the range $\overline{\mathrm{r}}=0.05 \ldots 1.0$ can be approximated to $10-3$ by the expression 
Then the formula (11) will be as

$$
(\pi / 2)(1+0,175 r / R)
$$

$$
r \sqrt{R} \frac{\pi}{2}\left(1+0,175 \frac{r}{R}\right)=\left.\frac{2}{3} \rho^{3 / 2}\right|_{\rho_{1(0)}} ^{R+r},
$$

where $\rho_{I(0)}$ is unknown inner radius of the ring, which provides the formation of the flanging radius portion (see Fig. 3).

Substitute in $(8,14)$ the limits of integration

$$
r \sqrt{R} \frac{\pi}{2}\left(1+0,175 \frac{r}{R}\right)=\frac{2}{3}\left[(R+r) \sqrt{(R+r)}-\rho_{1(0)} \sqrt{\rho_{1}}\right],
$$

and let us resolve the equality (15) with respect to $\rho_{1}$

$$
\rho_{1(0)}=\left[(R+r) \sqrt{(R+r)}-\frac{3}{4} \pi r \sqrt{R}\left(1+0,175 \frac{r}{R}\right)\right]^{2 / 3}
$$

To determine the radius of hole for flanging, we use the volume equality of the elementary sections: the cylindrical and the initial flat ring for it

$$
2 \pi \mathrm{s} R d h=2 \pi s_{0} \rho d \rho
$$

After the conversion and integration of both sides with appropriate limits, we obtain a formula for determining the diameter of the hole in flare

$$
d_{h}=2\left[\left(\frac{D_{f l}}{2}+r\right)^{3 / 2}-\frac{3 \pi r}{4} \sqrt{\frac{D_{f l}}{2}} \cdot\left(1+0,175 \frac{2 r}{D_{f l}}\right)-\frac{3}{2} h \sqrt{\frac{D_{f l}}{2}}\right]^{2 / 3},
$$

where $d$ is the diameter and $r$ is the radius of rounding refers to the middle surface of the flanging, and $h$ is the height of the cylindrical part of the side (here $h=H-\left(s_{0}+r_{\text {rond }}\right)-$ see Fig. 3).

For the radius of the mid-surface $r / D_{f l}=0.05$, the formula (18) is converted to

$$
d_{h}=2\left[\left(\frac{D_{f l}}{2}+r\right)^{3 / 2}-\frac{3}{2} \sqrt{\frac{D_{f l}}{2}} \cdot\left(\frac{\pi}{2} r+h\right)\right]^{2 / 3}
$$

For the purpose of comparative assessment calculations of apertures diameters under flanging on formulas $(1-4)$ and $(18,19)$ were carried out. The following parameters were used in the calculations (see Fig. 3):

- the relative thickness $-s_{0} / D_{f l}$ within $2-5 \%$;

- the relative height of flanging - $H / D_{f l}$ within $15-25 \%$.

- the relative radius of curvature $-r / D_{f l}$ within $10-20 \%$.

The obtained results showed that at the relative height of the flanging up to $15-20 \%$, the discrepancy of these calculations between the known and proposed formulas do not exceed $3 \%$. However, already at a relative height of $25 \%$, the discrepancy is $9 \%$.

Currently, technology development and tooling design is performed on a computer using appropriate applications [11-14].

As stated, parametric modeling allows combining the automation of calculations with the implementation of their results in graphical construction. The following is a solution of automation problem of the main technological parameters calculation and construction of the tool for flanging round holes with the use of CAD system is presented.

Fig. 4 represents a sketch of the flanged part, which is practically a diagram for constructing a parametric model using the variables window, which allows automating the display of changes in both geometric and basic technological parameters during the product shaping. 


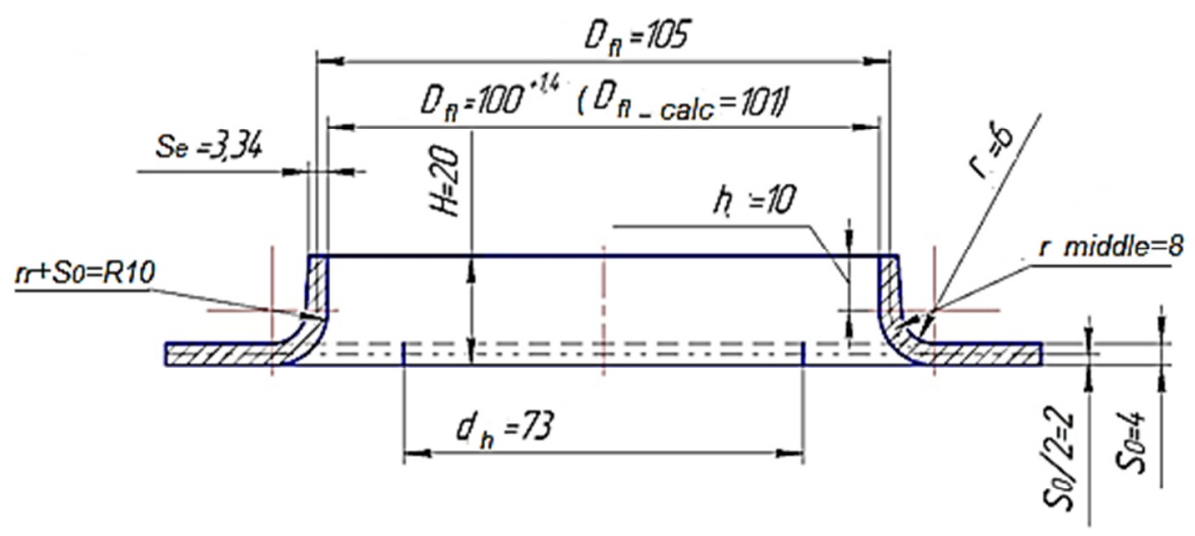

Fig. 4. Flanged workpiece sketch

When the sketch is created, the fragment document will be selected. The constructions are executed with the inclusion of the parametric mode required to activate the variable Window, presented in the form of a table. In this case, the dimensions are displayed through the Setup window in the dimension Variables section. However, for variables calculation formulas are required (expressions in CAD system terminology) and their further transfer to the dimension Variables section, it is provided to use the variables of the main section. The variables in this section do not directly control the parameters of the created sketch. Variables of the main section allow editing calculation formulas, assigning their names (in the English alphabet), moving them from one line to another, performing calculations that are not related to the sketch, drawing or model.

The formula (18) in order to simplify its processing by the calculation module of the application is divided into three components with the assignment of symbols $S 1 \_1, S 1_{-} 2$ and $S 1 \_3$

$$
\begin{gathered}
S 1 \_1=(D / 2+r) 3 / 2 \\
S 1 \_2=3 / 4 \cdot \pi \cdot(D / 2) 1 / 2 \cdot(1+0,175 \cdot(2 \cdot r) / D) \\
S 1 \_3=(3 / 2) h \cdot(D / 2) 1 / 2
\end{gathered}
$$

Now the formula (18) has acquired the form

$$
D h=2 \cdot\left(S 1 \_1-S 1 \_2-S 1 \_3\right) 2 / 3
$$

In accordance with all mentioned things above, the main section presents the calculation formulas and the results of their calculations. One of the main technological parameters is the force $P$ required for flanging. Approximately it can be found by the formula [2]

where $\sigma_{\mathrm{B}}$ - is the tensile strength.

$$
P=1,5 \pi(D-D h) s_{0} \sigma_{\mathrm{B}},
$$

The strength of the equipment required to perform the operation can be found from the ratio [2]

$$
P_{f l}=1,25 \ldots 1,5 \cdot P
$$

The flanging coefficient $C_{f l}$ is one of the determining factors of the correctness of the selected parameters [15]

$$
C_{f l}=D_{h} / D_{f l}
$$

When changes to the dimensions of the flanged workpiece and other data in the main section of the variables window is made, CAD system automatically recalculates the required parameters and displays them graphically in all forms of documents: fragments, drawings, models of both parts and assembly units. 


\section{Results}

The results of technological parameters calculations are the basis for the tooling construction. As mentioned above, CAD system provides the transfer of data (variable values) in associative mode from one file to another: here - to control the shape and size of the tool and other parts in the created stamp. Fig. 5 shows the scheme of the typical flanging stamp construction in the section. Here are the main details explaining the design of the stamp. The stamp works as follows. Sheet blank 1 with a hole for flanging is installed on the clamp 4 with fixing holes on the catcher 3 , mounted on the punch 3 . The upper flat part of the clamp 4 in the initial position is usually at the level with the flat end part of the punch. After turning on the press, the matrix 5 moves down, presses the workpiece 1 to the clamp together with the punch 2 deforms it and at the end of the working stroke forms into the product 6 . Next, the matrix is returned to its original position, and the product 6 is removed from the matrix by the ejector 7 .

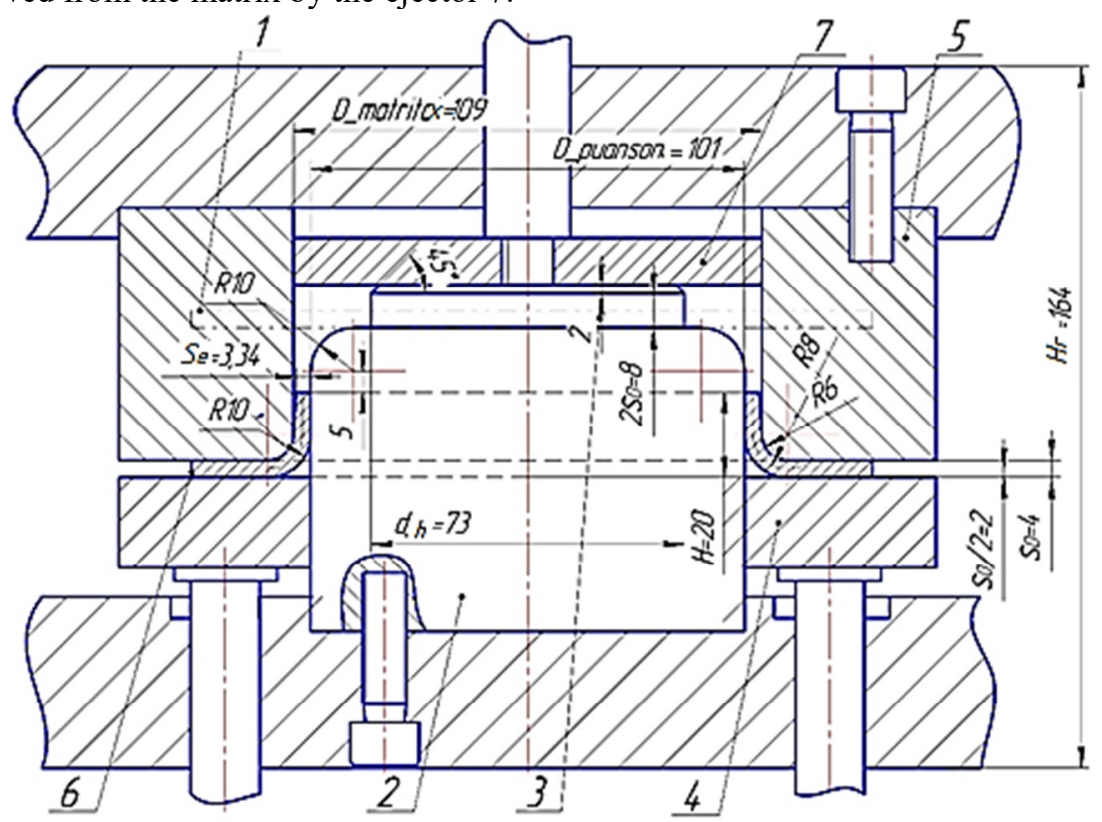

Fig. 5. Scheme of the stamp standard construction for flanging (1-blank; 2-punch; 3-catcher; 4-clamp; 5-matrix; 6 - product; 7 - ejector)

The transfer of data from the Main section to the Dimension section is carried out by analogy with the Variable window of the workpiece sketch. At the same time, the variant of calculating the approximate meaning of the stroke of the press slider $H P$ is presented, to which the upper part of the stamp with a matrix and the ejector is attached

$$
H P=\left(H-s_{0}\right)+r_{\text {puanson }}+2 \cdot s_{0}+2+10,
$$

where $H$ - is the height of the side, $s_{0}$-is the initial thickness of the material, $r_{\text {puanson }}-$ is the radius of rounding of the working punch edge. The remaining dimensions are defined structurally in accordance with Fig. 5.

In CAD system, when building in parametric mode, dimensions are divided into Control and Information: the first controls a geometric object, which controls the second. An example of the Information size is the closed height of the stamp $\left(H_{r}=164\right.$ - see Fig. 5). 


\section{Discussion and conclusions}

1. Given formulas increase the calculating accuracy of the workpiece hole diameter for flanging.

2. The accepted scheme of deformations distribution and the condition of constancy will allow constructing three-dimensional model of a product that gives the chance to use joint methods for the analysis of its stress-strain state in a process of deformation and an estimation of durability of prepared products.

3. Developed and implemented algorithm in CAD system of the flanged parts automatic sketch and determine the main technological parameters.

4. The generated scheme standard of stamp for flanging is constructed, the file which is associated in the associative mode with the sketch detail that provides automatic display change parameters of the tool.

5. Implementation of the article results will simplify the development of technological and constructed documentation; reduce the cost of production preparation.

\section{References}

1. K. Mori, S. Nishijima, C. J. Tan. International Journal of Machine Tools and Manufacture, 49(10), 767-772 (2009) DOI: 10.1016/j.ijmachtools.2009.04.005

2. H. Kaderov, S. Kireev et al. IOP Conference Series: Earth and Environmental Science, 403, 1-7 (2019) DOI: 10.1088/1755-1315/403/1/012088

3. M.P. Pereira, W. Yan et al. Wear, 268(11-12), 1275-1284 (2010) DOI: 10.1016/j.wear.2010.01.020

4. P. Rulli, R. Marquis. Forensic Science International, 301, 445.e1-445.e8 (2019) DOI: 10.1016/j.forsciint.2019.03.043

5. J. Zheng, X. Shu et al. Thin-Walled Structures, 127, 135-144 (2018) DOI: 10.1016/j.tws.2018.01.040

6. J. Choi, B. Choi et al. Applied Thermal Engineering, 1285, 159-172 (2018) DOI: 10.1016/j.applthermaleng.2017.09.001

7. L.I. Besong, J. Buhl, M. Bambach. International Journal of Mechanical Sciences, 164, 105143 (2019)

8. H-S. Lin, C-Y. Lee et al. International Journal of Machine Tools and Manufacture, 47(1), 168-174 (2007) DOI: 10.1016/j.ijmachtools.2006.02.002

9. R. Hino, T. Kuzukawa et al. Procedia Engineering, 207, 1928-1933 (2017) DOI: 10.1016/j.proeng.2017.10.963

10. H-S. Lin, C-W. Tung. Journal of Machine Tools and Manufacture, 47(14), 2133-2139 (2007) DOI: 10.1016/j.ijmachtools.2007.04.010

11. F-H. Yeh, M-T. Wu et al. Journal of Machine Tools and Manufacture, 47(12-13), 1854-1863 (2007) DOI: 10.1016/j.ijmachtools.2007.04.002

12. Z. Qian, L. Shi et al. Procedia Engineering, 207, 1272-1277 (2017) DOI: 10.1016/j.proeng.2017.10.882

13. A. Govik, L. Nilsson, R. Moshfegh. Journal of Materials Processing Technology, 212(7), 1453-1462 (2012) DOI: 10.1016/j.jmatprotec.2012.02.012

14. W. Dong, Y. Li, Q. Lin. et al. Procedia Manufacturing, 15, 961-968 (2018) DOI: 10.1016/j.promfg.2018.07.399

15. S.K. Paul, M. Mukherjee, S. Kundu. et al. Computational Materials Science, 8915, 189-197 (2014) DOI: 10.1016/j.commatsci.2014.03.040 